\title{
L'ASTED, dix ans après : bilan et prospective
} ASTED after 10 years: evaluation and perspective La ASTED diez años después: resumen y perspectivas

\section{Denis Rousseau et Hubert Perron}

Volume 30, numéro 3, juillet-septembre 1984

URI : https://id.erudit.org/iderudit/1053541ar

DOI : https://doi.org/10.7202/1053541ar

Aller au sommaire du numéro

\section{Éditeur(s)}

Association pour l'avancement des sciences et des techniques de la documentation (ASTED)

ISSN

0315-2340 (imprimé)

2291-8949 (numérique)

Découvrir la revue

Citer cet article

Rousseau, D. \& Perron, H. (1984). L'ASTED, dix ans après : bilan et prospective. Documentation et bibliothèques, 30(3), 71-86. https://doi.org/10.7202/1053541ar
Résumé de l'article

Les auteurs font ressortir les gestes positifs posés par l'Association pour l'avancement des sciences et des techniques de la documentation (ASTED) depuis sa création en 1974 et signalent ses faiblesses et ses omissions. Quatre domaines retiennent leur attention : la formation professionnelle et continue, la coordination des services documentaires, leur " promotion » et la vie de l'Association.
Tous droits réservés (c) Association pour l'avancement des sciences et des techniques de la documentation (ASTED), 1984
Ce document est protégé par la loi sur le droit d'auteur. L’utilisation des services d'Érudit (y compris la reproduction) est assujettie à sa politique d'utilisation que vous pouvez consulter en ligne.

https://apropos.erudit.org/fr/usagers/politique-dutilisation/ 


\title{
L'ASTED, dix ans après: bilan et prospective
}

\author{
Denis Rousseau* \\ Office de la langue français (OLF) \\ Montréal \\ Hubert Perron* \\ Université du Québec \\ Montréal
}

Les auteurs font ressortir les gestes positifs posés par l'Association pour l'avancement des sciences et des techniques de la documentation (ASTED) depuis sa création en 1974 et signalent ses faiblesses et ses omissions. Quatre domaines retiennent leur attention: la formation professionnelle et continue, la coordination des services documentaires, leur "promotion» et la vie de l'Association.

\section{ASTED after 10 years: evaluation and perspective}

The authors bring out the positive actions of the ASTED since its creation in 1974, and also point out its weaknesses and omissions. Four fields hold their attention: professional and adult formation, coordination of documentation services, their "promotion" and the activities within the association.

\section{La ASTED diez años después: resumen y perspectivas}

Los autores resumen las actividades de los diez años de la existencia de la ASTED. Cuatro temas llaman la atención: la formación profesional y educación de adultos, la coordinación de servicios documentales, la promoción de servicios documentales y la vida de la Asociación.
Le premier janvier 1974 naissait I'ASTED. L'Association pour l'avancement des sciences et des techniques de la documentation succédait ainsi à l'Association canadienne des bibliothécaires de langue française (ACBLF) fondée quelques dizaines d'années plus tôt, en 1943.

Les fondateurs de l'ACBLF avaient établi la nature de l'Association et délimité son champ d'action en fonction des réalités professionnelles, sociales et politiques du temps. Mais au début des années soixante-dix, le besoin d'une redéfinition des objectifs et d'un élargissement des structures et du champ d'action se faisait sentir. Aussi, afin de procéder à cette mise à jour, les responsables de l'ACBLF formèrent-ils la Commission de revision des objectifs et des structures dont le mandat était de revoir la nature et le fonctionnement de
I'Association. Des recommandations de cette commission, présidée par Jean-Rémi Brault ${ }^{1}$, naquit I'ASTED.

Mil neuf cent quatre-vingt quatre marque donc le dixième anniversaire d'existence de l'ASTED. Pour souligner cet événement, le comité de rédaction de Documentation et bibliothèques nous a invités à dresser le bilan de l'action de l'Association au cours de ses dix années d'existence.

Dès le départ, deux évidences se sont imposées à nous. Il fallait tout d'abord dresser une sorte d'historique de I'ASTED. Mais devant l'ampleur de cette tâche, il nous est apparu que les réflexions et les analyses pourraient facilement se perdre dans les méandres des multiples interventions de I'ASTED sur la scène de la bibliothéconomie

\footnotetext{
* M. Rousseau était, jusqu'à récemment, responsable de la bibliothèque de l'OLF et $M$. Perron est Directeur des bibliothèques de I'UOAM.

Les auteurs remercient ceux qui ont participé activement à l'élaboration de ce texte, MM. Réal Bosa, Onil Dupuis, YvonAndré Lacroix et Marcel Lajeunesse.
}

1 Association canadienne des bibliothécaires de langue française, Rapport de la Commission de revision des objectifs et des structures, Montréal, I'Association, 1972, 96, 6p. 
québécoise et canadienne. Aussi, avons-nous choisi de ne faire ressortir que les faits et les interventions les plus marquants de l'Association.

Nous nous sommes, d'autre part, refusés à ne présenter qu'une simple énumération des événements: notre souci allant au-delà de la chronique, nous avons voulu connaître également la perception qu'ont les membres de leur Association, ce qu'ils en pensent et aussi ce qu'ils en attendent. D'où la dimension prospective que nous avons ajoutée à ce bilan.

Trois possibilités s'offraient à nous.

1) Dresser ce bilan à partir de nos propres recherches historiques et de notre perception de l'Association, cette dernière pouvant être enrichie des perceptions de notre entourage professionnel immédiat recueillies au hasard des conversations et des rencontres.

Nous n'avons pas retenu cette démarche parce qu'elle prêtait le flanc à une trop grande personnalisation du texte et à une vision peutêtre trop individualisée du rôle joué par l'ASTED depuis dix ans.

2) Faire un sondage en bonne et due forme. La méthode du sondage est lente d'application (mise au point du questionnaire, temps de réponse, compilation des résultats, etc.) et très coûteuse en temps. De plus, elle ne favorise pas nécessairement l'expression spontanée des personnes interrogées. Nous avons donc repoussé cette deuxième possibilité.

3) Consulter directement un certain nombre de membres de l'Association selon un plan préétabli.

Cette méthode permettait une dépersonnalisation de l'exercice en élargissant l'éventail des opinions. Elle favorisait aussi une expression plus spontanée des personnes consultées par le jeu de la dynamique de groupe. C'est l'option que nous avons retenue.

\section{La consultation}

Nous avons choisi d'inviter à une rencontre plusieurs membres de l'ASTED qui ont été, et qui sont encore, actifs au sein de l'Association. Réunis en ateliers, puis en séance plénière, ils ont étudié quatre thèmes qui recouvrent à peu près toutes les activités de l'Association: la formation du personnel des bibliothèques et des centres de documentation (formation professionnelle et formation continue, ce qui inclut les publications), la coordination des services documentaires, leur "promotion» et, finalement, les membres de I'ASTED et la vie de leur Association. Pour ce faire, nous avons pu compter sur la précieuse collaboration de quatre "recherchistes" et animateurs: Réal Bosa, Marcel Lajeunesse, Yvon-André Lacroix et Onil Dupuis.

Un bilan comporte toujours deux volets: un actif et un passif. L'opération consistait donc à faire ressortir les gestes positifs posés par I'ASTED au cours de ses dix années d'existence (et il y en a eu beaucoup) mais aussi à signaler, sans complaisance, ses faiblesses, ses oublis, ses omissions. Le tout devrait servir à orienter l'action future de I'ASTED. Cette dimension de prospective est importante et utile: ne dit-on pas volontiers que le passé est garant de l'avenir?

\section{Formation du personnel des bibliothèques et des centres de documentation}

\section{Techniciens en documentation}

La formation de techniciens en documentation a mobilisé beaucoup d'énergie au sein de l'ASTED. Lors de leur arrivée sur le marché du travail, ces spécialistes ont eu à lutter pour conquérir leur place dans les bibliothèques, les bibliothécaires n'étant pas tous sensibilisés à l'importance, voire à la richesse de leur apport.

Très tôt, les techniciens ont senti le besoin de se regrouper afin de pouvoir discuter de leurs problèmes et des conditions d'exercice de leur profession. Reconnaissant ce besoin, l'ASTED créa le Comité des bibliotechniciens ${ }^{2}$ qui devint le centre nerveux de leur activité et de leur recherche d'une place au soleil dans la bibliothéconomie québécoise.

Cependant, ils avaient également besoin d'asseoir solidement les bases de leur formation. Par le biais de son Comité de bibliotechnique, I'ASTED a décidé de s'impliquer dans ce dossier en intervenant directement auprès du ministère de l'Éducation et auprès des collèges d'enseignement général et professionnel qui dispensaient les cours en techniques de la documentation.

De 1974 à 1978 surtout, on a beaucoup discuté du contenu du nouveau programme de techniques de la documentation, le Comité de bibliotechnique collaborant d'ailleurs étroitement à son élaboration. L'ASTED est intervenue auprès du ministère de I'Éducation, et plus particulièrement auprès de la Direction générale de l'enseignement collégial (DGEC) afin qu'on restreigne à quatre le nombre d'établissements dispensant les cours en la matière au lieu des neuf alors existants. Cette démarche était rendue nécessaire par la pléthore de diplômés issus des cégeps en regard des besoins du marché.
2. Il est bon de distinguer le Comité des bibliotechniciens, animé par des bibliotechniciens et des techniciens, dont le but était de permettre à ces derniers d'échanger leurs expériences et leurs problèmes, du Comité de bibliotechnique (maintenant connu sous le nom de Comité des techniques de la documentation) animé par des bibliothécaires et des techniciens et axé principalement sur la formation de ces derniers. Le Comité de la formation professionnelle des techniciens en documentation, pour sa part, vise à l'établissement d'un programme universitaire de premier cycle en bibliothéconomie accessible aux techniciens. 
L'ASTED n'a malheureusement pas obtenu gain de cause puisque sept cégeps dispensent encore ces cours.

Puis s'est dessiné peu à peu le problème du plan de carrière des techniciens. Longtemps en filigrane des discussions entourant le nouveau programme, ce problème jaillit finalement de la conscience des principaux intéressés eux-mêmes, les techniciens, puis des bibliothécaires, surtout des bibliothécaires-employeurs, lesquels rendent compte peu à peu de l'importance pour ces employés de faire carrière dans leur profession et d'y progresser normalement.

On se rappellera qu'en 1970, I'École de bibliothéconomie de l'Université de Montréal instaurait le programme de deuxième cycle en bibliothéconomie. Elle suivait en cela une pratique généralisée en Amérique du Nord puisque la majorité des écoles de bibliothéconomie limitaient déjà leurs cours au seul niveau de la maîtrise. L'année suivante, on voyait arriver sur le marché du travail les premiers détenteurs d'une maîtrise en bibliothéconomie et, aussi, les derniers bacheliers.

A partir de cette date, l'écart entre ces deux niveaux de formation fit réfléchir bon nombre de membres de la profession. $Y$ avait-il place pour une catégorie intermédiaire de diplômés? $\mathrm{Ne}$ pouvait-on pas réinstaurer le programme de premier cycle en bibliothéconomie tout en prenant soin de le rendre accessible aux techniciens déjà sur le marché du travail et désireux de compléter leur formation, ouvrant ainsi vers le haut leur perspective de carrière?

Au terme de plusieurs rencontres et après l'étude de diverses hypothèses, l'ASTED décida, en 1981, de confier à son Comité de la formation professionnelle des techniciens en documentation le mandat d'étudier, de manière comparative, le programme de techniques de la documentation et celui de bibliothéconomie et d'élaborer un projet de programme de l'Université de Montréal en vue de l'adoption de ce projet. Jusqu'ici, le projet n'a pas encore été adopté, certaines difficultés techniques ayant été soulevées par l'Université de Montréal.

\section{Bibliothécaires}

Si le dossier de la formation des techniciens a mobilisé l'ASTED, il en va un peu différemment de celui de la formation des bibliothécaires. Depuis sa création, l'Association n'a pas manifesté d'intérêt particulier pour leur formation universitaire si ce n'est de leur formation continue, ce dont nous traiterons plus loin. Rappelons que le dernier congrès annuel traitant de la formation du per- sonnel des bibliothèques remonte à 1970. C'était donc du temps de l'Association canadienne des bibliothécaires de langue française!

Deux événements majeurs survenus aux écoles de bibliothéconomie des universités McGill et de Montréal auraient pourtant dû intéresser grandement I'ASTED en tant qu'association professionnelle nationale : entre 1974 et 1983, les deux écoles obtenaient tout d'abord le renouvellement de leur agrément de l'American Library Association; puis I'École de l'Université de Montréal élabora un tout nouveau programme de maîtrise tandis que celle de McGill remaniait le sien. L'Association a délégué quelques-uns de ses membres aux divers sous-comités chargés de préparer le renouvellement de l'agrément de l'École de bibliothéconomie de I'Université de Montréal et Documentation et bibliothèques a consacré son éditorial de septembre $1974^{3}$ à l'agrément. Mais là s'arrêta l'intérêt de l'ASTED pour ces questions.

Aquoi attribuer ce demi-silence de l'Association sur la formation universitaire d'une bonne partie de ses membres? Y a-t-il eu entente, plus ou moins tacite, avec la Corporation des bibliothécaires professionnels du Québec (CBPQ) à l'effet de laisser ce dossier à cette dernière? Plusieurs des personnes consultées opinent dans ce sens. Toutefois, rien dans les rapports et les textes officiels ne permet de l'affirmer avec certitude. Cette attitude générale de l'ASTED surprend puisque la formation est aussi de son ressort.

Par contre, ce manque d'intérêt évident et regrettable de I'ASTED pour la formation des bibliothécaires pourrait être, disent certains, le reflet ou la conséquence du manque d'intérêt de l'École elle-même pour la profession telle qu'elle se pratique au Québec. Il est un fait que l'École de bibliothéconomie de I'Université de Montréal s'ouvre de plus en plus à la dimension internationale (échange de professeurs avec l'École nationale supérieure des bibliothèques de France, participation à la mise en place d'un programme de maîtrise à l'Université de Dakar (Sénégal), contributions de plus en plus nombreuses des professeurs à des revues étrangères, participation très active à l'Association internationale des écoles de sciences de l'information (AIESI), accueil de nombreux étudiants étrangers). Ce rayonnement est louable aux yeux de tous, mais doit-il se faire au détriment de la profession elle-même, de ses besoins réels et concrets au sein de la société québécoise? L'internationalisme, d'accord, mais l'École de bibliothéconomie est avant tout une école nationale, affirment certains, une école qui devrait répondre en priorité aux besoins d'ici.

Quoi qu'il en soit des opinions sur cette question, un fait demeure : I'ASTED doit s'impliquer davantage dans la formation universitaire des bibliothé- 
caires par des contacts officiels, étroits et soutenus, avec les écoles de bibliothéconomie. Tous s'accordent à le réclamer. La poursuite même de ses objectifs ne peut que l'y entraîner. Et nous ajouterions qu'il y va aussi de l'intérêt des écoles de nouer des liens solides avec l'association représentant les professionnels en exercice.

À l'automne 1975, Gérard Martin, alors chargé d'évaluer l'action de l'ASTED en regard de ses objectifs d'origine, deplorait le manque d'intérêt de I'ASTED vis-à-vis du programme de l'École de bibliothéconomie ${ }^{4}$. Force est de constater que son regret conserve toute son actualité.

Notons enfin que les personnes consultées ne nient pas à la CBPQ le droit d'intervenir dans ce dossier: bien au contraire, cette dernière doit percevoir une action dans ce domaine comme étant une de ses priorités. Cela n'exclut pas pour autant I'ASTED qui a, elle aussi, une grande responsabilité en cette matière. Les deux associations pourraient d'ailleurs y trouver un terrain propice à une collaboration efficace.

Quelle forme pourrait prendre cette participation de l'ASTED à l'action des écoles professionnelles? Des consultations régulières et suivies, nous dirions même «institutionnalisées", paraissent essentielles. L'ASTED étant au coeur de l'exercice de la profession et, de ce fait, généralement bien informée de l'évolution du marché, elle constitue un interlocuteur de première importance pour les écoles.

D'autres avenues peuvent également être explorées, telles la formation continue (dont nous traiterons au chapitre suivant), la mise sur pied de programmes de recherche et la préparation de publications.

\section{Formation professionnelle continue}

S'il est un secteur d'activité qui mérite une attention particulière de la part de l'Association, c'est bien celui de la formation professionnelle continue, surtout en cette période de bouleversements profonds des activités documentaires comme l'automatisation massive, la présence sur la scène documentaire de plusieurs groupes de spécialistes de l'information, le militantisme syndical accrû, l'élaboration de nouvelles méthodes de gestion et l'éclatement de la notion même de bibliothèque. Les bibliothécaires ne sont plus seuls sur la scène documentaire, ils doivent gérer la croissance avec des budgets à base-zéro, accepter de travailler avec celui qu'on osa même nommer "l'homme de l'année", l'ordinateur, et encore intégrer d'autres types et d'autres supports d'information (micro-éditions, œuvres d'art, vidéotextes, logiciels, etc.) dans leurs préoccupations.

Au cours de la décennie, I'ASTED a organisé plusieurs activités de formation continue à l'intention de ses membres, mais elle a éprouvé certaines difficultés à offrir avec régularité et cohérence un véritable programme d'ateliers, de journées d'étude et de sessions de formation. De 1974 à 1982 (à l'exception des années 1977 et 1978 qui furent pratiquement stériles en cette matière), I'ASTED a parrainé, en moyenne, 1,5 stage par année. C'est peu compte tenu des besoins de la profession.

Mentionnons néanmoins, à titre de réalisations heureuses, deux colloques organisés par le Comité des relations internationales en 1979 et en 1982 qui ont d'ailleurs mené à des publications: Documentation, information et développement international/Libraries, Information and International Development $(1981)^{5}$ et Information et documentation: I'attente des pays en développement/The Expectations of Developing Countries (1982) 6 . Un séminaire sur la micro-informatique organisé en collaboration avec Informalog connut un véritable succès: réalisé une première fois le 27 octobre 1982, il dut être repris quatre fois en février et mars 1983 tant l'intérêt était grand. Mentionnons également le stage intensif sur le vocabulaire libre en indexation qui donna lieu, lui aussi, à une publication en 1978 .

A plusieurs reprises, l'ASTED s'est jointe à ses partenaires sur la scène documentaire québécoise pour organiser des activités de formation. En novembre 1981, par exemple, elle unissait ses efforts à ceux de l'École de bibliothéconomie de I'Université de Montréal et à ceux de l'AUPELF pour la tenue d'un colloque sur les attentes de pays en développement; une publication, dont nous avons parlé plus tôt, devait en émaner. Les 28 et 29 janvier 1982, le Comité de perfectionnement de l'ASTED, encore en collaboration avec l'École de bibliothéconomie, présentait une session de formation sur les bases de données bibliographiques. Le 2 avril de la même année, l'ASTED se joignait à la CBPQ, à l'École de bibliothéconomie et à l'Association canadienne des sciences de I'information (ACSI) afin d'offrir une journée d'étude sur l'informatique en tant que véhicule ou témoin de l'information documentalre. On se rappellera également les quelques colloques organisés conjointement avec la Canadian Library Association (CLA), dont celui des 24 et 25 octobre 1978 portant sur les systèmes et réseaux de biblio-
4. "Rapport du comité ad hoc chargé de l'étude des recommandations de la Commission de révision des objectifs et des structures", Nouvelles de l'ASTED, (octobre 1975), 5.

5. Actes du colloque organisé par le Comité des relations internationales de l'ASTED, en collaboration avec la Canadian Library Association, Ottawa, les 20 et 21 juin 1979. Montréal, ASTED, 1981, $78 \mathrm{p}$.
6. Textes des conférences du colloque des 16 et 17 novembre 1981. Montréal, ASTED, 1982, 89, 74 p.

7. Raymond Blais et Yves Courrier, Vocabulaire libre en indexation. Montréal, ASTED, 1978, $180 \mathrm{p}$ 
thèques; l'organisation et la tenue de ces colloques font d'ailleurs l'objet d'une entente entre l'ASTED et la CLA.

On sait que la CBPO offre également à ses membres plusieurs activités de formation. Les personnes consultées sont d'avis que les deux programmes parallèles de formation constituent une perte de temps, d'énergie et d'argent. Les deux associations ne pourraient-elles pas mettre en commun leurs ressources humaines et financières afin d'offrir à l'ensemble de la profession un programme unique de formation continue mettant ainsi fin à la répétition, à la redondance et à la dispersion des efforts? Ce serait là consolider la collaboration déjà amorcée.

Nous savons tous cependant qu'il faut plus que l'information en capsule d'une ou deux journées de formation pour vraiment faire le tour de certaines réalités comme celle, par exemple, de l'automatisation des services documentaires! Des programmes cohérents de recyclage (entendons des cours) s'imposent donc et l'ASTED pourrait prendre l'initiative de négocier avec les écoles de bibliothéconomie, de pair avec la CBPQ, le cadre, le contenu et les modalités de réalisation de tels programmes. Cette mesure est d'autant plus impérieuse que les besoins sont pressants et rien n'est plus naturel que de se tourner vers les écoles professionnelles pour la réalisation d'un tel projet puisqu'on y trouve les ressources pédagogiques.

Au chapitre de la formation continue, donc, plusieurs réalisations heureuses et une collaboration amorcée et réussie avec I'Ėcole de bibliothéconomie de l'Université de Montréal et la CBPO en particulier. On note cependant l'absence d'un programme-cadre de formation professionnelle continue intégrant les divers types de formation: cours de recyclage, colloques, conférences, journées d'étude, séminaires. L'ASTED s'est occupée du dossier de la formation continue avec succès; il lui resterait à aller un peu plus loin et à procéder un peu moins à la pièce. Elle répondrait ainsi pleinement à un des buts fixés par la Commission de révision des objectifs et des structures:

Développer un programme de formation professionnelle permanente pour le personnel des bibliothèques, des centres de documentation et d'information ${ }^{8}$.

\section{Publications}

Publier, c'est «faire connaître au public», c'est «annoncer publiquement», nous dit le Petit Robert. L'ASTED, par ses publications, se révèle à son public. Les publications sont la preuve tangible de la vitalité de l'Association; elles témoignent de ses préoccupations, de ses orientations et de l'intérêt de ses membres pour la promotion et la défense de leurs idées et contribuent à l'avancement de la discipline, objet même de l'existence de I'Association.

Afin d'affirmer son rôle de leader au sein de la bibliothéconomie nord-américaine de langue française, I'ASTED s'était vu fixer comme objectif de "publier une revue et tous les ouvrages utiles à l'exercice de la profession "9

Cet objectif, I'ASTED l'a rempli en bonne partie de 1974 à 1977 en effet, cinq ouvrages furent publiés en 1974-1975, six en 1975-1976 et quatre en 1976-1977 dont Livre, bibliothèque et culture québécoise; mélanges offerts à Edmond Desrochers, s.j. ${ }^{10}$.

L'ASTED avait reconnu l'importance du secteur des publications puisque, dès septembre 1974, elle retenait les services à plein temps d'un responsable des publications afin de développer un programme de publication et de coordonner l'ensemble de sa production. Des difficultés financières majeures l'obligèrent cependant à renoncer à cette prestation deux années plus tard. La production s'en ressentit: de 1977-1978 à 1982, I'ASTED n'a publié en moyenne que 2,33 ouvrages par année, dont les très populaires Règles de catalogage anglo-américaines ${ }^{11}$. On pourrait ajouter que la hausse vertigineuse des coûts d'impression qui a frappé le monde de l'édition au milieu des années soixante-dix n'a pas aidé l'ASTED, forcée qu'elle était déjà de fonctionner dans de strictes limites budgétaires, pas plus d'ailleurs que l'empressement plus que modéré des membres à acquérir les publications de leur propre Association!

Les thèmes ou domaines couverts par les publications sont des plus variés, allant de la littérature enfantine aux règles de catalogage en passant par la philosophie de la bibliothéconomie et l'acquisition des documents, sans oublier la promotion des bibliothèques publiques et le vocabulaire libre en indexation.

Une entente intervenue entre l'École de bibliothéconomie de l'Université de Montréal et l'ASTED en 1975 fit de cette dernière l'éditeur et le diffuseur exclusif des publications de l'École. C'est ainsi qu'on inaugura les collections "Publications de l'École de bibliothéconomie» et "Documentation en diagonale" afin d'y regrouper les publications émanant des travaux et des recherches menés à l'École. Bien engagée avec la publication
8. Association canadienne des bibliothécaires de langue française, Rapport..., p. 53.

9. Ibid., p. 50

10. Georges-A. Chartrand, éd., Livre, bibliothèque et culture québécoise; mélanges offerts à Edmond Desrochers, $s$. j. Montréal, ASTED, 1977, 2 v
11. Règles de catalogage anglo-américaines, $2 \mathrm{e}$ édition. Rédigées en collaboration par The American Library Association, The British Library, le Comité canadien de catalogage, The Library Association, The Library of Congress; Version française d'après l'édition nord-américaine (1973), établie sous la direction de Paule Rolland-Thomas avec la collaboration de Pierre Deslauriers, Montréal, ASTED, 1980, XXIV, 938 p. 
de plusieurs titres, cette entente aurait actuellement besoin d'être revitalisée si l'on veut éviter qu'elle ne tombe dans I'oubli!

Soulignons que I'Association a maintenu la parution régulière de son bulletin de nouvelles et de sa revue Documentation et bibliothèques. La qualité matérielle de cette dernière a malheureusement tendance à diminuer, notent les personnes consultées, ce qui est perçu comme un affaiblissement de I'ASTED puisque Documentation et bibliothèques est devenue, au fil des années, son produit de marque. Sabrer dans le budget de cette revue, dans cet instrument de formation continue par excellence, c'est couper dans le vif puisque Documentation et bibliothèques est probablement le meilleur ambassadeur de l'Association, la preuve tangible de sa permanence et de sa vitalité. Cette revue dont la crédibilité n'est plus à faire et qui se méritait, en 1978, le Library Periodical Award 1977 décerné par la H.W. Wilson Company «pour son exceptionnelle contribution à la bibliothéconomie et aux sciences de l'information canadiennes-françaises et au développement de la bibliothéconomie dans les pays d'expression française du monde entier "12 requiert une attention particulière de la part des administrateurs de l'Association.

Quant aux Nouvelles, on en déplore l'assèchement et la froideur des chroniques. Où sont les polémiques d'un passé pas si lointain? Les leaders d'opinion se seraient-ils tus? Ou auraient-ils perdu le goût de s'exprimer? Quels problèmes concrets agitent la profession? Les Nouvelles ne répondraient que faiblement à ces préoccupations, farcies qu'elles sont de comptes rendus de réunions et d'annonces d'événements. On n'y débat plus des idées, on y consigne plutôt des faits et on y étale des rapports et des communiqués. Cela est bien mais les lecteurs espéreraient davantage : ils aimeraient y trouver un peu plus de vie, voire des controverses. Une revitalisation de cet organe d'animation de l'Association serait donc souhaitable. Et ce n'est pas la seule responsabilité des rédacteurs des Nouvelles. Les membres ont à leur disposition un important moyen de communication pour exposer leurs idées, leurs problèmes et proposer leurs solutions.

\section{Recherche}

Le rapport Brault formulait un objectif on ne peut plus louable: "Susciter et financer des recherches concernant la bibliothéconomie, la documentation et l'information ${ }^{13}$. Et on y suggérait que I'ASTED se dote d'un service de recherche chargé d'intensifier et d'activer l'évolution de la profession.

\section{L'Association n'a jamais donné suite à ce dernier}

souhait; elle n'a pas cependant complètement délaissé la recherche pour autant bien que sa fiche, à ce chapitre, ne soit pas très remplie! Le Comité de la recherche, dont Gérard Martin déplorait l'inactivité en 1975, s'est surtout borné à attribuer les bourses de recherche offertes par I'ASTED et ses commanditaires à des étudiants en bibliothéconomie et en techniques de la documentation. De ce fait, l'ASTED reconnaît davantage les recherches qu'elle ne les suscite.

Nous devons pourtant considérer Livre, bibliothèque et culture québécoise, dont nous avons parlé plus haut, comme le plus vaste bilan historique et descriptif jamais entrepris par le monde de la bibliothéconomie québécoise: plus de quarante auteurs et 840 pages de texte. Le passé de l'Association étant maintenant bien connu et les assises de la profession clairement cernées, nous attendons les recherches qui nous guideront dans l'avenir.

La bibliothéconomie québécoise est originale en ce sens qu'elle ne peut être considérée comme entièrement française sans pour autant être assimilée à la bibliothéconomie américaine dont elle subit pourtant l'influence : recherche de son originalité et implications pratiques pour le monde documentaire québécois, voilà de quoi inspirer un programme de recherche pour plusieurs années à venir!

II nous semble que s'offre ici le plus beau champ possible de collaboration avec les écoles de bibliothéconomie, puisque divers groupes de recherche y sont à l'œuvre. Une coordination des travaux de ces chercheurs et des besoins de la profession exprimés par l'ASTED serait profitable aux deux parties: rentabilisation du travail des premiers, amélioration de la qualité d'exercice de la seconde. Les étapes? D'abord, s'entendre sur les modalités de la collaboration, puis identifier les secteurs prioritaires de recherche et de développement et, enfin, mettre sur pied des projets spécifiques tout en recherchant les sources de financement. De plus, le protocole de publication conclu entre I'ASTED et I'École de bibliothéconomie de I'Université de Montréal constitue un stimulant à la recherche puisqu'il peut devenir un utile canal de diffusion des travaux des chercheurs.

Dans cet ordre d'idées, I'ASTED décidait tout récemment de verser à son fonds de recherche et de développement le surplus budgétaire découlant de la tenue de la 48 e Conférence générale de I'IFLA (International Federation of Library Associations and Institutions). On se rappellera que le Comité d'organisation de cette conférence, avec l'appui de son Comité d'honneur, avait plutôt recommandé de créer, avec ce surplus, une fondation indépendante de I'ASTED et vouée exclusivement à la recherche en bibliothéconomie et en
12. Documentation et bibliothèques, vol. 24 , no 3 (septembre 1978), 157
13. Association canadienne des bibliothécaires de langue française, Rapport ..., p. 51 
sciences de l'information. La sollicitation de subventions et de dons pour des projets spécifiques en aurait été grandement facilitée. L'ASTED a préféré miser sur son propre fonds de recherche privant peut-être ainsi le secteur de la recherche de certains apports financiers puisqu'elle-même reçoit déjà une subvention en tant qu'organisme culturel. Néanmoins, des sommes importantes seront désormais disponibles.

La véritable recherche, il faut l'admettre, a peu préoccupé l'ASTED jusqu'à maintenant. Un vigoureux coup de barre s'impose d'autant plus que les quatre éléments nécessaires à la réalisation d'un programme de recherche sont déjà réunis: les institutions (ASTED et écoles), les chercheurs (praticiens, professeurs, étudiants), le canal de diffusion (entente ASTED / École)et le financement. Les sommes nécessaires peuvent être obtenues auprès d'organismes de subvention ou à même le fonds de recherche et de développement de l'Association. II manque donc peu de choses pour que le programme soit lancé.

\section{Coordination des services documentaires}

La coordination! Que voilà un bien grand mot ! La langue française l'a compris et c'est pourquoi elle s'avère plutôt riche pour exprimer cette idée de partage et d'interconnexion : concertation, coopération, rationalisation, planification. Aquelques nuances près, ces termes impliquent tous la notion d'échange, de partage, d'action commune selon un plan précis. Voilà définie la coordination. II reste à y ajouter un objectif: I'atteinte d'une fin déterminée. En termes documentaires, cela revient à mettre en commun un certain nombre de ressources (documents, personnel et budgets) selon des modalités acceptées de tous afin d'en maximiser l'utilisation. C'est la gestion rationnelle du disparate intégré dans un tout!

Ce concept de coordination est relativement nouveau dans la documentation professionnelle. Deux facteurs principaux l'ont imposé à notre entendement : I'explosion documentaire-expression galvaudée, s'il en est une, mais néanmoins réelle-qui a pesé lourd dans la balance de l'échange de la documentation puisque plus personne ni aucun organisme ne peut se permettre de tout acquérir, même dans un seul domaine, et le tarissement des ressources financières, lequel exerça des pressions similaires sur le désir (!) et la volonté de collaboration des institutions.

C'est donc au début des années soixante-dix que la documentation professionnelle en vint à se nourrir à la mamelle de la coordination. Les écrits de la première heure ont coïncidé avec certains grands événements, tels la formation, aux États-
Unis, de la National Commission on Libraries and Information Science (1970) et la Conférence générale de I'Unesco sur le NATIS (National Information Systems) en 1974. Ajoutons la mise sur pied, par I'IFLA, de deux vastes programmes d'information documentaire: le Contrôle bibliographique universel (CBU) et la Disponibilité universelle des publications (DUP) On eut également, à Washington, la conférence générale de l'IFLA sur la planification nationale et internationale en 1974 et, dans la même foulée, celle de Montréal, en 1982, autour du thème des réseaux.

Ce vaste courant international, associé aux facteurs énumérés plus haut, a trouvé des échos au sein de la profession et de l'ASTED. Depuis une décennie, en effet, certains thèmes ont fait couler beaucoup d'encre et mobilisé quantité d'énergies. Mentionnons, entre autres, "l'implantation d'un réseau québécois de traitement informatisé des données catalographiques... depuis 1973, la préoccupation majeure des responsables du développement des bibliothèques québécoises"14. De nombreuses études, émanant tantôt de la profession, tantôt de firmes-conseil, ont fait l'objet de rapports présentés au gouvernement (projet CIDBEQ, Centre d'information documentaire des bibliothèques d'enseignement du Québec, 1974; rapport CERBIQ, c'est-à-dire du Comité d'étude d'un réseau de bibliothèques québécoises en 1978; rapport du Centre technique d'évaluation des bibliothèques universitaires québécoises (CTBU) en 1978, etc.).

L'idée de coordination s'est rapidement étendue à l'ensemble des ressources et des activités des bibliothèques. Trois événements majeurs, auxquels I'ASTED a été liée, retiendront plus particulièrement notre attention: la Rencontre sur la bibliothéconomie québécoise (1975), la Table ronde sur la planification des services documentaires québécois (1978) organisée à l'initiative même de I'ASTED et la 48e Conférence générale de I'IFLA (1982).

Du 29 au 31 janvier 1975 eût lieu ce qu'il est convenu d'appeler la Rencontre sur la bibliothéconomie québécoise dont l'aboutissement principal fut un accord de principe sur la création «d'un organisme d'étude chargé d'élaborer les modalités propres à établir la coordination de toutes les bibliothèques du Québec ${ }^{15}$. On voulait attribuer trois champs d'étude au nouvel organisme: établissement d'une superstructure de la bibliothéconomie québécoise, organisation d'un réseau québécois d'information documentaire et mise sur pied d'un ou de consortiums de services. Cet organisme d'étude, qu'on aurait nommé Comité d'étude sur la coordination des bibliothèques, a failli voir le jour comme en fait foi la correspondance échangée entre les organisateurs de la Rencontre
14. Création d'un serveur québécois dans le domaine des banques de données catalographiques, S.1., s.n., septeribre 1983, p. 2
15. Rencontre sur la bibliothéconomie québécoise, 29-31 janvier 1975, Manoir St-Castin, Lac Beauport, S. 1., La Rencontre. 1975, p. 6. 
et le ministre des Affaires culturelles du temps, Jean-Paul L'Allier. Le texte de l'arrêté-en-conseil créant ce Comité avait même été rédigé. En avril 1976, le projet fut temporairement ajourné par le ministre L'Allier lui-même qui allégua la préparation, alors en cours, de la politique du livre. Puis, officiellement, il n'en fut plus question et les promoteurs du projet mirent fin à leurs pressions. L'idée aura donc duré un an et quelques mois. Seuls résultats tangibles: une étude conjointe ASTED / CBPO sur les périodiques québécois en bibliothéconomie ${ }^{16}$ et le protocole d'édition et de diffusion conclu entre l'École de bibliothéconomie de I'Université de Montréal et I'ASTED, protocole dont il fut question précédemment.

Le défi de la coordination de l'ensemble des services documentaires était à nouveau relevé en 1978 alors que I'ASTED organisait une Table ronde sur la planification des services documentaires québécois. On voulait, encore une fois, en faire un moyen de pression sur les instances politiques afin qu'elles "prennent l'initiative d'établir, à brève échéance, une politique et des mécanismes pour une planification québécoise des services documentaires" 17 . On voulait également "sensibiliser les milieux de la documentation et l'ensemble de la population aux avantages d'une telle politique ${ }^{18}$.

L'entreprise était de taille : elle s'appuyait sur un dossier complet, élaboré par des bibliothécaires d'expérience, avec la participation de plusieurs :ninistres du gouvernement du Québec et la collaboration de spécialistes venus du Canada, des États-Unis et du Vénézuéla. C'est le 5 mai 1978 qu'eut lieu cette rencontre tant attendue entre les représentants de la profession et le gouvernement. L'apport plus que précieux des spécialistes étrangers, basé sur leur propre expérience, contribua grandement à éclairer le débat. Au dire des personnes consultées, cette Table ronde fut un geste sans précédent dans I'histoire de l'ASTED tant par son ampleur que par la précision de son organisation et la notoriété des personnes réunies.

Les suites ne furent malheureusement pas à la hauteur des efforts déployés. Il y eut, bien sûr, des promesses de la part du pouvoir politique mais à peu près rien de concret n'en découla. De son côté, I'ASTED revient à la charge en 1979, lors de la consultation gouvernementale autour du livre vert sur la politique scientifique ${ }^{19}$. On ne manqua pas de préciser que les structures de planification à mettre en place pour l'organisation des services documentaires englobaient, bien sûr, le secteur de l'information scientifique et technique. L'Association remit d'ailleurs un mémoire, rédigé conjointement par les différentes associations de bibliothéconomie, au ministre d'état au Développemet culturel, Camille Laurin, dans lequel elle précisait sa position.

D'où vient que cet événement de la Table ronde soit tombé à plat? L'enjeu était-il trop grand? Que s'est-il donc passé?

Comme il était coutume à cette époque au Québec-et c'était là une véritable tendance sociale - on comptait sur le gouvernement pour apporter des solutions à tous les problèmes. On lui confiait donc des dossiers et puis on attendait. C'est ce que fit l'ASTED. Et mal lui en prit puisque la profession attend encore!

La Table ronde fut un immense effort de sensibilisation du gouvernement au problème de la coordination des services documentaires. On lui déblaya le terrain puis on attendit. Au dire de certains participants, il aurait peut-être mieux valu proposer des solutions concrètes et manifester le désir de les réaliser soi-même avec l'appui et la collaboration de l'État. En d'autres termes, ne pas lâcher le dossier et garder l'initiative de sa réalisation. Faille, donc, au niveau de la stratégie.

Les membres de I'ASTED que nous avons consultés sont également d'avis qu'il y eut faiblesse au niveau du suivi à donner à une telle proposition. Des pressions soutenues, un lobbying intensif auprès des ministres impliqués et une vigoureuse campagne de presse à l'intérieur même de la profession auraient peut-être donné des résultats différents.

Nous touchons sans doute là au talon d'Achille de cette Table ronde: les membres de I'ASTED, autres que ceuxqui y furent directement impliqués, étaient-ils vendus à l'idée de la planification et de la coordination? Éprouvaient-ils, face à ce problème, le même sentiment d'urgence que les organisateurs de la Table ronde? II semble que non, l'expectative plus que l'implication personnelle étant le dénominateur commun.

II est sans doute facile, diront certains, de formuler des remarques à quelques années de l'événement. Certes, le temps a permis une analyse. Mais ce manque de suivi d'un dossier et cette absence d'implication de la base de la profession ne sont pas des cas uniques dans l'histoire de I'ASTED. Ne serait-il pas souhaitable que, collectivement, nous en prenions conscience afin de corriger notre tir à l'avenir?
16. Une revue scientifique de bibliothéconomie au Québec? Rapport du Comité mixte ASTED / CBPQ sur les périodiques québécois en bibliothéconomie, Montréal, 31 août 1976, $67 \mathrm{p}$. La principale recommandation de cette étude se lisait comme suit : «Qu une revue scientifique unique de bibliothéconomie constituée à partir des éléments appropriés de Documentation et bibliothèques et d'Argus soit mise sur pied" (p. 65). Cette étude n'a jamais eu de suites; ses recommandations conservent pourtant toute leur actualité.
17. Extrait du dossier déposé lors de la rencontre avec les ministres du gouvernement du Québec.

18. Ibid.

19. Québec, Ministère d'état au Développement culturel. Pour une politique québécoise de la recherche scientifique, Québec, Éditeur officiel du Québec, 1979, VI, 222 p. 
Un troisième événement d'importance, qui souligne l'intérêt de l'ASTED pour la coordination, fut la tenue de la 48 e Conférence générale de l'IFLAà Montréal du 22 au 28 août 1982 sous le thème "Les réseaux". C'est à l'invitation de I'ASTED, en effet, que le Bureau de direction de I'IFLA acceptait, en 1978, de tenir à Montréal sa 48 e Conférence générale. Le Comité d'organisation, mis sur pied par I'ASTED dès 1979, travailla d'arrache-pied pendant trois ans à faire de cette conférence une réussite, mobilisant les efforts d'une centaine de membres de la profession. Les délibérations qui réunirent plus de 1900 participants de 73 pays, portèrent principalement sur la constitution et le fonctionnement des réseaux documentaires à l'échelle nationale et internationale.

Véritable succès au plan de l'organisation et de la participation, il est encore trop tôt cependant pour juger de l'impact de ce rassemblement puisqu'un événement d'une telle ampleur ne peut avoir d'effets réels qu'à moyen et à long terme sur le discours et surtout sur l'action professionnelle. Néanmoins, les propos actuels de nos spécialistes de la coordination et de la planification sont indubitablement teintés des couleurs de cet événement.

Nous ne pouvons terminer ce tour d'horizon de l'activité de l'ASTED dans le secteur de la coordination sans souligner sa participation, par le biais de ses représentants, à diverses activités de coordination et de planification, telles la révision des règlements de prêt entre bibliothèques (en collaboration avec la CLA) et l'édition française des Règles de catalogage anglo-américaines.

Si la coordination doit demeurer une des préoccupations essentielles de l'Association - et les membres consultés le croient fermement - il serait peut-être opportun, voire impérieux, que l'ASTED se dote d'une structure (comité ou autre) capable de gérer ce dossier, d'en assurer le suivi et d'y impliquer les membres de la profession. Car c'est là ce qui semble avoir dramatiquement fait défaut: I'ASTED n'a pas donné suite, de façon systématique, aux projets qu'elle avait elle-même mis de l'avant et les membresn'ont pas vraiment accroché à ces grands dossiers parce qu'ils n'y étaient probablement pas suffisamment sensibilisés.

Ce comité ou cet organisme, formé de membres ayant un mandat de quelques années, pourrait établir un programme d'action à moyen et à long terme qui soit dégagé des impératifs des diverses présidences et qui puisse assurer une action continue des projets de vaste envergure.

L'ASTED, ont dit certains, aurait pu s'impliquer davantage dans les grands programmes de coordination mis de l'avant par des organismes internationaux tel I'IFLA, à savoir le Contrôle bibliographique universel (CBU) et I'Accès universel aux publications (AUP). II n'est peut-être pas trop tard pour le faire. Mais, chose certaine, une constatation s'impose : en dépit de quelques grands succès, I'ASTED aurait avantage à réviser son mode d'intervention dans ce secteur maintenant névralgique de la documentation et à se doter d'une structure douée d'une certaine continuité. Comme l'heure des réalisations a sonné, elle pourrait même prendre l'initiative de proposer des modèles de coordination en présentant diverses approches: globales, sectorielles ou régionales (programme de coopération en matière d'acquisitions, consortium de services, entrepôt commun).

\section{Promotion des services documentaires}

Les bibliothèques et centres de documentation ne sont pas, par définition, des établissements qui tournent à vide : ils n'existent que par et pour les usagers actuels et éventuels. Á quoi servirait, en effet, d'empiler les témoins physiques de la connaissance s'ils ne sont bons qu'à meubler des rayons et à remplir des classeurs ? La Commission de révision des objectifs et des structures avait voulu prémunir l'Association naissante contre le danger du repli sur soi en lui fixant comme but « de promouvoir l'excellence des services et du personnel des bibliothèques, des centres de documentation et des centres d'information ${ }^{20}$. De ce but, découlait l'objectif de «faire connaître l'existence et les services de l'Association et de ses membres par une publicité intelligente, imaginative et bien orchestrée»21.

Que fit l'Association? Avant de répondre à cette question, il importe de faire une distinction. L'ASTED, estimons-nous, a deux publics à rejoindre: le public interne constitué essentiellement de ses membres et le public externe qui comprend les professionnels du monde de la documentation (ou, si l'on veut, ses membres potentiels), les média et le grand public (c'est-à-dire les utilisateurs actuels et possibles des services documentaires) et, enfin, les gouvernements. Auprès de ces publics, I'ASTED fait deux types de promotion: tout d'abord sa propre promotion (sa nature, ses objectifs, ses structures, ses réalisations), puis la promotion des services qu'elle-même ou que ses membres rendent ou peuvent rendre à l'ensemble de la collectivité. II va sans dire que les moyens choisis pour faire passer les messages varient considérablement selon le public auquel I'ASTED s'adresse.

\section{Public interne}

Le public interne, à savoir les membres, est le plus facile à identifier et à cerner et aussi le plus facile à rejoindre. Il est, somme toute, réceptif et il attend qu'on l'informe, voire qu'on le stimule! 
Assez paradoxalement d'ailleurs, il s'implique s'il se sent en cause. Ainsi, par exemple, il sera disposé à œuvrer au sein d'un comité dont le domaine le concerne.

C'est donc le premier public auquel I'ASTED s'est adressé. Depuis dix ans, I'Association a consacré beaucoup d'énergie, de temps, d'argent et d'imagination à faire sa propre promotion auprès de ses membres afin d'inciter tous et chacun à la reconnaître et à l'appuyer : promotion de ses buts et objectifs, mise en évidence de ses réalisations et de ses interventions, énumération des diverses facettes de son activité (comités et sections, stages, congrès, etc.) où chacun peut se réaliser et avantages qui découlent d'une participation à titre de membre (échange constant d'informations, réception de publications telles les Nouvelles et la revue Documentation et bibliothèques, abattement fiscal). Comme exemple de ce type de promotion, on peut mentionner le tout dernier dépliant d'information de l'Association qui s'intitule L'ASTED et vous. Cette forme de publicité vise surtout à démontrer aux membres que leur Association est vivante, dynamique et qu'elle veille à leurs intérêts.

A ce chapitre de la promotion interne, on peut rattacher les actions menées par les comités et les sections, actions qui prennent la forme soit de journées d'étude sur le comment et le pourquoi du marketing et des relations publiques, soit d'articles dans Documentation et bibliothèques sur les mêmes sujets, le tout susceptible d'aider les membres à agir sur leur public externe et, plus particulièrement, sur le grand public. Ajoutons aussi les dépliants d'information, les lettres de sollicitation, les stands dans les congrès et les catalogues de publications, tous des moyens de promotion que l'ASTED maîtrise finalement assez bien. Les résultats ont-ils été à la hauteur des attentes? II semble que non puisqu'on constate un réel désengagement des membres en dépit de tous les efforts déployés. La difficulté de trouver des candidats pour ouvrer au sein de certains comités et du Bureau en est un bel exemple.

\section{Public externe}

S'il est une catégorie de public que l'ASTED a tenté d'intéresser, c'est bien celle des membres de la profession qui n'ont pas encore joint ses rangs. Partant du principe que plus une association compte de membres, plus elle a de chances d'être forte, I'ASTED a beaucoup misé sur le recrutement, lequel aura été, il faut bien l'admettre, une de ses préoccupations principales, sinon sa préoccupation majeure, au cours de ses dix années d'existence. Que de stratégies ont été élaborées pour recueillir l'adhésion soit des membres démissionnaires, soit de membres potentiels appartenant aux différentes disciplines du livre, de la documentation, de l'information et des communications, tels les libraires, les archivistes et les informaticiens! Encore là, les résultats n'auront pas été à la mesure des efforts déployés : le chapitre suivant, qui traitera des fluctuations du nombre de membres, montrera que la réponse a été décevante.

II est intéressant de noter que le comité des relations publiques, d'abord voué presque exclusivement à la conception et à la réalisation de programmes de relations publiques visant le grand public, est vite devenu le Comité des relations publiques et du recrutement. A-t-on procédé à ce regroupement par souci de planification des actions de publicité ou parce qu'on aurait relégué au second plan, plus ou moins consciemment, les actions auprès de ce public tant la préoccupation du recrutement était devenue généralisée au sein de I'ASTED ?

II semble, au dire des personnes consultées et à la lumière des événements, qu'il faille malheureusement retenir la deuxième hypothèse. Le grand public, tel qu'il est convenu de l'appeler, et les média - puisque les deux sont intimement liés auront donc été les partenaires négligés de I'ASTED.

Non pas que tout soit négatif à ce chapitre : il ya eu, certes, quelques temps forts, mais les réalisations furent tellement sporadiques que la désolation s'empare de l'esprit le plus optimiste! Mentionnons tout d'abord la publication du dossier publicitaire destiné à faire connaître les bibliothèques publiques puis la semaine de promotion des ouvrages de référence au Québec organisée en collaboration avec le ministère des Affaires culturelles du Québec, laquelle a fourni l'occasion de publier un cahier spécial du journal Le Devoir en mars $1980^{22}$. Soulignons également la campagne menée auprès des journaux lors de la fermeture de la bibliothèque de la Commission des écoles catholiques de Montréal en 1977. Ajoutons aussi les conférences Aegidius-Fauteux et un autre cahier spécial du journal Le Devoir publié le 21 août 1982 à l'occasion de la 48e Conférence générale de I'IFLA. Ce dernier cahier portait sur l'état des bibliothèques au Québec. Si nous mentionnons enfin l'existence du concours Marie-Claire-Daveluy ouvert aux jeunes auteurs de quinze à vingt ans et l'attribution du prix AlvineBélisle décerné à la meilleure œuvre de l'année en littérature de jeunesse, nous avons à peu près fait le tour des grandes interventions de l'ASTED auprès du grand public.

Pourtant, les services offerts quotidiennement par l'ASTED et ses membres intéressent tous les citoyens : qu'on parle de lecture publique, d'accès à l'information ou de disponibilité de la docu-

22. "L'accès à l'information: les années 80 ; place à la communication individuelle», Le Devoir (cahier spécial), 15 mars $1980,10 \mathrm{p}$ 
mentation, tous s'accordent à voir dans ces produits certaines des composantes les plus essentielles de la société actuelle. L'information sous toutes ses formes est devenue une denrée précieuse, voire rentable,autant pour les hommes d'affaires et les politiciens que pour les étudiants et les simples citoyens : tout le monde s'informe, se renseigne, s'arrache même les données les plus à jour afin d'en faire son profit. L'ombre de l'ASTED se profile-t-elle sur ce monde avide de savoir? L'association a-t-elle présenté ses produits et ses services dans le langage courant ? A-t-elle mobilisé les média écrits et électroniques afin de trouver en eux ces indispensables multiplicateurs de l'information et rejoindre ainsi son public ? II semble, disent nos informateurs, qu'il faille plutôt répondre par la négative.

Dans sa publicité, I'ASTED s'est beaucoup préoccupée du recrutement de nouveaux membres. Mais on a peut-être sous-estimé l'impact que peuvent avoir des actions et des services connus du grand public et un nom qui passe régulièrement dans les média sur le nombre d'adhésions. L'interaction, dans ce domaine, est réelle. Se faire connaître peut être un geste de survie pour une association et se traduire souvent par un accroissement réel du nombre de membres.

Il convient toutefois de nuancer nos propos par une remarque d'ordre technique. Même si les structures de I'ASTED ne seront étudiées qu'au chapitre suivant, nous pouvons affirmer, à ce stade-ci, que le fonctionnement actuel de l'Association, et tout particulièrement son mode décisionnel, ne permet pas une intervention rapide sur la place publique suite à des événements nécessitant une action immédiate. De plus, l'ASTED n'a pas réussi à clarifier le rôle et les fonctions de ses divers agents de relations publiques, à savoir le président, le directeur général, le Comité des relations publiques et, à l'occasion, les spécialistes en relations publiques. En outre, le secrétariat ne semble posséder ni les pouvoirs ni les ressources humaines et financières qui lui permettraient d'agir rapidement en situation d'urgence (consultation-éclair des administrateurs, envoi de télégrammes, émission de communiqués de presse, organisation de conférences de presse, et autres). Annonce-t-on l'abolition du Service des bibliothèques publiques, c'est sur-le-champ qu'il faut réagir. Le gouvernement publie-t-il un projet de politique sur la lecture publique, il faudrait illico faire connaître la position de l'Association aux journalistes et, par ricochet, au grand public. C'est ainsi que se bâtit la réputation de dynamisme et de vitalité d'un groupe.

Ceci nous amène à traiter d'une autre catégorie de public externe que I'ASTED doit rejoindre, les gouvernements. Ces derniers, qu'ils soient de niveau municipal, provincial ou fédéral, élaborent généralement longtemps à l'avance leurs politiques, leurs projets de lois et leurs règlements avant de les rendre publics. C'est également un secret de Polichinelle qu'ils n'agissent généralement qu'en réaction aux pressions du milieu. Et d'où viennent ces pressions? De groupes de pression, telle I'ASTED. Nous plongeons, vous l'avez deviné, dans la jungle du lobbying.

Le Rapport Brault disait de I'ASTED qu'elle doit inspirer la législation et promouvoir les intérêts respectifs des bibliothèques, des centres de documentation et des centres d' information auprès des gouvernements concernés 233 II allait même jusqu'à recommander la mise sur pied d'un comité permanent chargé de . conseiller les autorités gouvernementales dans la préparation, l'élaboration et la rédaction des lois qui concernent les membres de l'Association et leurs activités professionnelles $" 24$

Le rôle de lobbyiste de l'ASTED n'est pas facile à cerner puisque, par définition, le lobbying peut se faire insinuant et très discret, empruntant les voies détournées des contacts personnels et utilisant des informateurs influents à des postes-clés. Mais il peut aussi devenir beaucoup plus officiel et prendre la forme de mémoires présentés au gouvernement, de résolutions adoptées en assemblée générale, de lettres officielles appuyant des demandes ou énonçant des prises de position.

L'ASTED a pratiqué ces deux formes de lobbying avec assez de succès puisqu'elle est devenue un interlocuteur écouté de l'État, atteignant en cela le deuxième but fixé dans le Rapport Brault : « Devenir, auprès du gouvernement québécois, le porteparole officiel et principal pour toutes les questions concernant les bibliothèques, les centres de documentation et les centres d'information ${ }^{25}$.

On a consulté I'ASTED, entre autres, lors de l'élaboration du plan quinquennal de développement des bibliothèques publiques, de la politique québécoise de l'information scientifique et technique et de la politique de la lecture. L'ASTED a également fait valoir son point de vue, à de multiples reprises, par le biais de lettres et de mémoires adressés au gouvernement, en son nom propre ou en collaboration avec d'autres organismes, la CBPQ et I'Association des bibliothécaires du Québec ( $A B Q$ ) par exemple, sur des sujets très variés, comme la formation des techniciens en documentation, la fermeture de bibliothèques, la tarification des services dans les bibliothèques publiques et le droit d'auteur. II reste que cette deuxième forme d'intervention (mémoires et lettres) a souvent constitué une réaction à des projets gouvernementaux ou même à des gestes déjà posés, plaçant ainsi l'ASTED sur la défensive.

Les prises de position de l'Association n'ont pas toujours été épousées par les gouvernements et 
on peut se demander si, fortement appuyées par l'ensemble de la profession et la presse, elles n'auraient pas donné, dans certains cas, des résultats différents. Car le lobbying comporte un troisième volet qui, malheureusement, a été trop négligé, celui de l'implication des membres et aussi des média dans une cause commune. Adresser une demande au gouvernement est une chose, mais le faire avec l'appui massif des membres et des média en est une autre. Plus une revendication recueille d'appuis, plus elle a de chances d'être entendue. C'est, comme nous l'avons déjà souligné, ce qui a dramatiquement fait défaut, par exemple, lors de la tenue de la Table ronde sur la planification des services documentaires québécois en 1978 et, plus récemment, lors du sommet socio-économique sur les communications.

Que dégager de ce chapitre sur l'action " promotionnelle » de I'ASTED ? D'abord la constatation que l'ASTED s'est beaucoup préoccupée de mousser son image auprès de ses clientèles interne et potentielle. On relève également quelques gestes publicitaires vraiment réussis destinés à sa clientèle externe, et plus particulièrement, au grand public. Quant aux divers paliers de gouvernements, ils ont reçu beaucoup de suggestions et de revendications de l'Association tant et si bien qu'on la considère comme un porte-parole digne de foi. On sait par contre que I'ASTED a souvent négligé ou omis de sensibiliser ses membres et les média aux grandes causes qu'elle a défendues. Il est pourtant important d'engager les membres à prolonger, par leur action individuelle, l'action collective de I'Association.

En somme, l'ASTED, dans ce domaine comme dans celui de la coordination, a souffert de l'absence d'un programme d'ensemble cohérent de publicité et de relations publiques incluant les divers publics. Son action trop ponctuelle a enregistré certaines réussites, d'ailleurs remarquées, mais aussi des échecs regrettables.

Toute intervention future aurait avantage à $s$ 'inscrire dans une politique globale qui fasse une large place à la sensibilisation du public interne, politique qui permette de définir clairement les objectifs poursuivis et qui identifie précisément les divers moyens d'action prévus auprès de chacun des publics. Car tout se tient dans ce domaine, les divers éléments s'influençant et agissant les uns sur les autres. Ce faisant, I'ASTED pourra peut-être, non seulement se contenter de réagir aux événements, mais prendre l'initiative de projets spécifiques et avoir une possibilité accrue de succès.

\section{Vie de l'Association}

Les chapitres précédents font état des réalisations de I'ASTED dans trois grands secteurs d'activités propres à une association professionnelle à vocation générale. A ce stade-ci, il nous apparaît approprié de nous interroger sur la vie même de notre regroupement : sa vitalité, son leadership, ses structures, sa raison d'être.

\section{Vouloir-vivre collectif}

Une association de nature volontaire, comme l'ont écrit les auteurs du Rapport Brault, repose sur le vouloir-vivre collectif de ses membres. A la fin de l'année 1983, l'ASTED comptait un peu plus de 800 membres, et ce dans un contexte de crise économique qui frappait plusieurs de ses membres, la plupart oeuvrant dans le secteur public. Que peut-on en déduire ? Sans doute que la majorité des membres de l'ASTED continuent à croire en sa mission. Cependant, il y a lieu de faire preuve de prudence.

De 1980 à 1983, le nombre des membres est passé de 1022 à 822 . On remarque également une forte baisse du nombre de techniciens (236 en 1980 par rapport à 123 en 1983). Un tel phénomène est contraire aux attentes exprimées lors de la création de l'ASTED, laquelle devait regrouper le plus grand nombre possible de spécialistes autres que les bibliothécaires. Par contre, pour la même période, le nombre de membres collectifs a légèrement augmenté $(202 / 210)$ comme celui des membres de l'extérieur du Québec (96/105) (Voir les tableaux 1, 2 et 3).

De ces quelques données, rien de très alarmant ne se dégage bien que la situation demeure inquiétante. On est en droit toutefois de s'interroger sur le bien-fondé des grands objectifs de regroupement des spécialistes de la documentation mis de I'avant par I'ASTED. Les énergies déployées n'ont pas produit les effets souhaités. Sur une période de dix ans, on assiste plutôt à un plafonnement.

Sans contester le souci primordial de l'ASTED de veiller à la constitution d'un front commun de la documentation et de travailler à contrer la dispersion des forces vives de la profession, elle a sans doute fait preuve d'un manque de réalisme et de sens pratique. Les tableaux précédents illustrent un revers évident et justifient une nouvelle orientation.

Dans ce contexte, le projet de Fédération québécoise de l'information documentaire (ROID) ne risque-t-il pas, faute d'analyse sur les aspirations des divers groupes à réunir dans le respect de leur individualité et faute d'études précises sur les objectifs et les champs souhaitables de mise en commun des ressources, de s'avérer un leurre? En définitive, nous croyons que pour trouver des solutions adaptées aux besoins, il est impérieux de bien définir ce que l'on attend de ce futur «regroupement» pour s'assurer de la réalisation de l'adéquation désirée entre les objectifs et les moyens. 


\section{Structures}

Les recommandations 3 à 17 du Rapport Brault concernent les moyens à prendre afin d'assurer l'atteinte des objectifs visés par l'ASTED ${ }^{26}$. Parmi ces recommandations, plusieurs se rapportent spécifiquement aux structures de l'Association. En 1975, le comité ad hoc, présidé par Gérard Martin et chargé de l'étude des recommandations de la Commission de révision des objectifs et des

Tableau 1 :

Evolution du nombre de membres de I'ASTED selon l'origine géographique, de 1977 à 1983

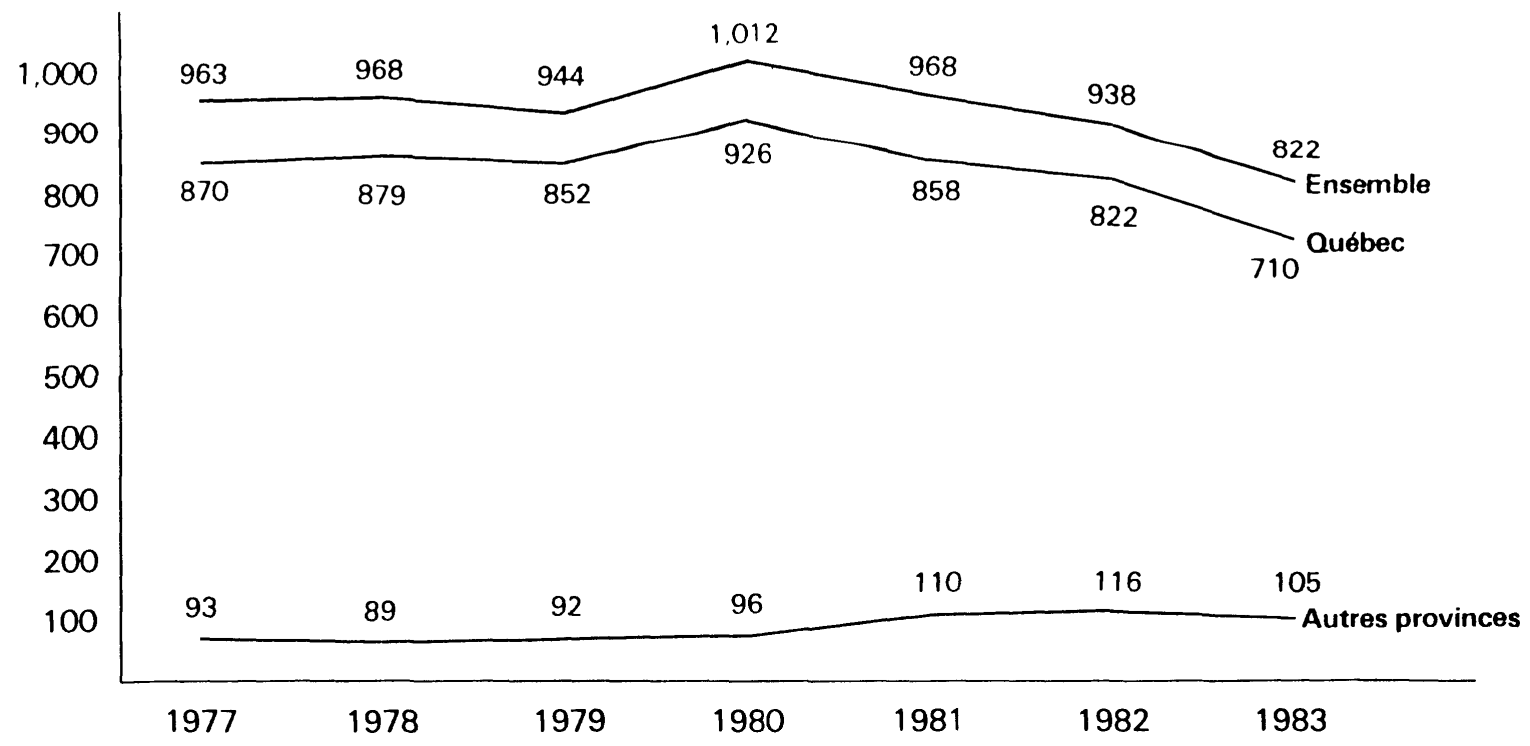

Tableau 2:

Evolution du nombre de membres collectifs et personnels de l'ASTED de 1977 à 1983

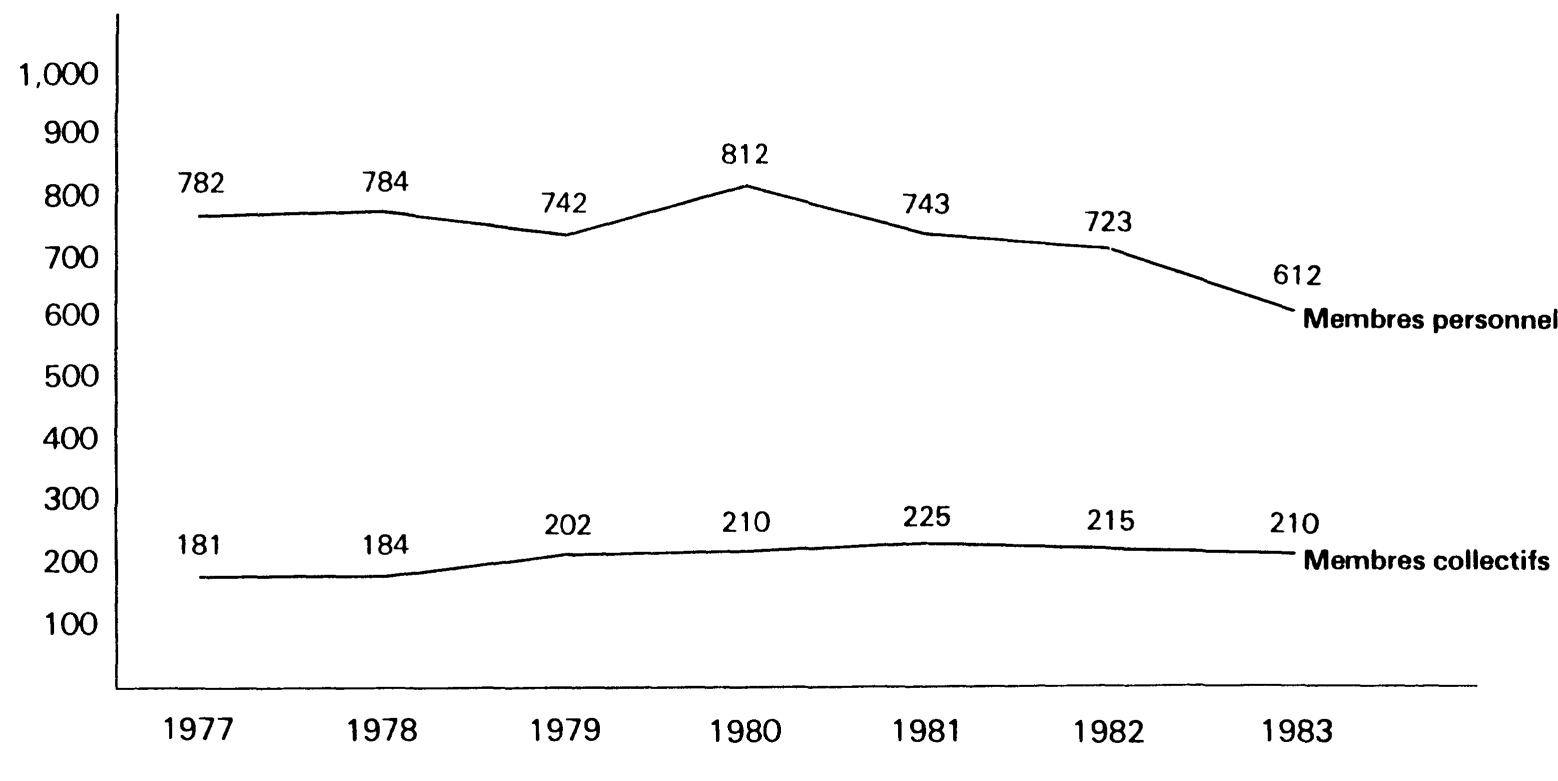


Tableau 3:

Membres individuels de l'ASTED selon les catégories professionnelles

\begin{tabular}{|c|c|c|c|c|}
\hline \multirow{2}{*}{ CATÉGORIE PROFESSIONNELLE } & \multicolumn{4}{|c|}{ ANNEEE } \\
\hline & $1980^{*}$ & 1981 & 1982 & 1983 \\
\hline $\begin{array}{l}\text { Analystes } \\
\text { Archivistes } \\
\text { Techniciens en documentation } \\
\text { Bibliothécaires } \\
\text { Documentalistes } \\
\text { Éditeurs } \\
\text { Libraires } \\
\text { Autres } \\
\text { Sans indication }\end{array}$ & $\begin{array}{r}4 \\
1 \\
236 \\
418 \\
19 \\
12 \\
10 \\
15 \\
97\end{array}$ & $\begin{array}{r}1 \\
4 \\
179 \\
369 \\
13 \\
- \\
- \\
31 \\
146\end{array}$ & $\begin{array}{r}2 \\
1 \\
156 \\
353 \\
20 \\
1 \\
-\quad \\
28 \\
162\end{array}$ & $\begin{array}{r}6 \\
3 \\
123 \\
350 \\
12 \\
- \\
\overline{2} \\
93\end{array}$ \\
\hline TOTAL & 812 & 743 & 723 & 612 \\
\hline
\end{tabular}

* 1980 à 1983 sont les seules années pour lesquelles les chiffres sont disponibles.

structures, deux ans après leur adoption, s'interrogeait déjà sur l'application faite par le Conseil d'administration des recommandations 11,12 et 13 relatives aux sections selon les types de bibliothèques et aux comités par types de formation des membres. Ce rapport insistait en même temps sur l'urgence de la création d'un comité de la constitution et des règlements et il traitait longuement du rôle essentiel d'un éventuel comité du plan'27.

Les structures administratives et les règlements de I'ASTED favorisent-ils, par leur souplesse et leur cohérence, l'engagement et l'active participation des membres notamment au sein des sections? L'existence même des sections taillées d'après les types de bibliothèques aurait-elle avantage à être remise en question? Favorise-t-elle ou empêchet-elle l'intégration des différents spécialistes de la documentation? Leur rôle se distingue-t-il suffisamment de celui des comités permanents et ad hoc?

C'est à ces interrogations, ainsi qu'à celles que nous avons soulevées plus haut, que I'ASTED entendait répondre en proposant, lors de sa dernière assemblée générale, l'abolition des sections et leur remplacement par des groupes d'intérêts:

Facilité de création, simplicité de fonctionnement, autonomie appréciable, ce sont là des facteurs qui devraient permettre aux groupes d'intérêts proposés de constituer pour les membres un cadre de regroupement et d'action mieux adapté à leurs désirs d'initiative et par conséquent susceptible de rendre l'Association plus féconde, et plus apte à jouer le rôle de leadership que ses membres voudraient la voir assumer ${ }^{28}$.
Il est cependant permis de se demander en quoi le mode de fonctionnement de ces groupes d'intérêts diffère de celui des sections et s'ils ne risquent pas de consacrer une part importante de leurs énergies à des préoccupations de nature administrative au détriment de leurs préoccupations professionnelles. Par ailleurs, la participation automatique au Bureau des présidents des groupes d'intérêts d'au moins vingt-cinq membres risque d'alourdir encore davantage le fonctionnement d'un Bureau qui compte déjà un nombre élevé d'administrateurs élus par l'ensemble des membres. II serait sûrement possible de mettre en place un mécanisme plus souple permettant aux groupes d'intérêts d'acheminer leurs préoccupations au Bureau. Ce mécanisme pourrait s'apparenter à celui en vigueur pour les comités. Les préoccupations des groupes d'intérêts seraientelles plus importantes et plus stables, et ainsi moins diverses et ponctuelles, que celles de comités comme celui des publications?

A propos du Bureau de l'ASTED, il est permis de s'interroger sur la représentativité réelle de ses membres en regard des intérêts professionnels de l'ensemble des membres. Force est de constater que le processus d'élections démocratiques, souhaité par le rapport Brault, a été remplacé, de fait, par des mises en candidature uniques aux différents postes et, en particulier, au poste de président. Si l'ampleur des responsabilités afférentes à ces postes effraie les candidats potentiels, n'y aurait-il pas lieu de repenser le mode d'élection et de proposer un mécanisme qui, par exemple, permettrait l'élection d'un groupe homogène ou d'une équipe? 


\section{Vitalité et leadership}

Un tel flottement, depuis dix ans, dans la recherche de structures adéquates - malgré la présence du comité de coordination qui a accompli un travail remarquable en ce qui a trait au rôle, au mandat et a la composition des comités, puis des réflexions et des travaux du comité d'orientation - révèle un malaise évident.

En dépit d'un bilan positif (mentionnons, entre autres, la Table ronde sur la planification des services documentaires québécois, l'organisation de la 48 e Conférence de l'IFLA, la participation de I'ASTED au Sommet socio-économique sur les communications, des colloques remarqués dans le domaine des relations internationales, des publications diverses dont la revue Documentation et bibliothèques), il est difficile de déceler un fil conducteur ou un programme d'action. On a plutôt l'impression d'assister à des coups d'éclat souvent sans lendemain. A l'exception des comités statutaires de l'Association, les activités des autres comités et sections sont empreintes d'improvisation, de fragmentation et de dispersion et ne constituent souvent que des actions ponctuelles.

En outre, les assemblées générales de l'Association et les rapports des diverses instances sont dépourvus de tout intérêt professionnel d'envergure et les congrès ne donnent lieu à aucun débat public sur les grandes questions du moment. Par surcroît, les assemblées générales des dernières années se sont terminées par des résolutions à caractère technique fournissant peu d'orientation. II faudrait s'en étonner!

L'ASTED réagit beaucoup face à toute espèce de projet de tiers, notamment face à ceux des gouvernements, mais elle agit peu. Les réflexions sur les structures tournent à vide, car elles ne s'inspirent pas d'un plan directeur étayé de programmes d'action à moyen terme. Déjà en 1975, Gérard Martin écrivait à ce sujet:

Avec le comité des finances, ce comité du plan, très bien décrit à la page 50 du Rapport Brault, serait l'organisme interne le plus important de I'ASTED, et il n'existe pas encore ...

Son rôle serait de définir et de tenir à jour une sorte de «plan directeur» non seulement concernant l'ASTED et ses activités, mais aussi un plan projeté sur l'avancement des sciences et des techniques de la documentation ainsi que sur le développement des services (quantités et qualités) offerts par les bibliothèques, centres de documentation et centres d'information, en partant de l'état actuel (voir l'avant-propos) en déterminant où nous avons l'intention d'être, où nous avons $l^{\prime}$ intention que la profession soit dans deux ans, cinq ans, dix ans d'ici. Aux olympiques, il n'y a pas de compétitions de piétinage ${ }^{29}$.

Ce comité du plan a finalement été formé. En fonction pendant un certain temps, il a cessé ses activités pour des raisons nébuleuses et sans avoir donné les résultats escomptés.

\section{Conclusion}

L'ASTED a été, et est encore, la principale association à caractère général capable de promouvoir la cause de la documentation et de défendre les intérêts du milieu des bibliothèques et des centres de documentation. Le bilan général de ses activités est, somme toute, positif: les nombreuses réalisations énumérées tout au long de cet article en témoignent.

Une question cependant doit être soulevée: I'ASTED pourra-t-elle demeurer cette associationcarrefour des préoccupations et des attentes du monde de la documentation et de l'information? On sait que flotte dans l'air depuis un certain temps un projet de regroupement des associations et organismes intéressés à la documentation. Ce projet, en dépit de son intérêt, est un foetus qui se présente mal. II est donc raisonnable de penser que, à court et à moyen terme tout au moins, I'ASTED pourrait demeurer ce lieu privilégié d'échange d'opinions et cette source principale d'initiatives capables de promouvoir la cause de la documentation.

Mais elle demeurera, et c'est là que la bât blesse, à la condition que des correctifs réels soient apportés à son activité et à son fonctionnement. Au cours de cette étude, nous avons signalé plusieurs faiblesses et carences qui ont souvent diminué, sinon miné, le rayonnement de ses gestes et de ses interventions: manque de suivi des dossiers, non-sensibilisation de l'ensemble de ses membres aux grandes causes qu'elle défendait, actions trop ponctuelles et lenteur de la prise de décision, lacunes qui pourraient être corrigées par un plan d'ensemble et à longue portée d'interventions et d'actions.

Lewis Carroll affirmait fort justement: «If you don't know where you are going, any road will get you there". L'absence de direction précise entraîne forcément une action sans orientation certaine. Aussi, un comité du plan repensé et mieux encadré, attelé à la tâche, exigeante, certes mais combien emballante, de définir la profession de demain, de tracer les grandes orientations et les gestes à poser en fonction des composantes de la profession et de la société serait, à notre avi, l'élément central susceptible d'assurer l'avenir de l'Association. 
L'ASTED a perdu le goût des débats publics que ce soit au niveau de son public interne ou de son public externe. Ces échanges permettraient de discuter des dossiers de nature générale, des dossiers, dirions-nous, "horizontaux»qui touchent tout le monde. Car il ne faudrait jamais l'oublier, ce qui différencie l'ASTED de beaucoup d'autres associations tant au Québec, qu'au Canada et qu'en Amérique du Nord, c'est qu'elle est, d'abord et avant tout, une association à caractère général. D'où l'importance de concentrer ses ressources sur des dossiers de même nature, lesquels présentent un intérêt soit pour l'ensemble des membres, soit pour le grand public ou les deux à la fois et nécessitent une continuité dans l'action assurée par une structure donnée. C'est là qu'entre en jeu le Comité du plan, le plus apte à réaliser cette importante forme d'activité de l'ASTED. Des exemples de dossiers de cette catégorie? Nous en avons déjà mentionné plusieurs: formation, recherche, coordination et publicité.

L'ASTED doit s'intéresser à d'autres questions plus spécifiques, lesquelles pourraient former un deuxième type d'activités: il s'agit des activités des divers groupes d'intérêts qui se répartissent par types de bibliothèques ou de personnes et qui reposent sur le vouloir-vivre d'un groupe. Ainsi pourrait-on retrouver, par exemple, le groupe des techniciens, le groupe scolaire et le groupe des sciences juridiques.

Les activités régulières de fonctionnement de I'ASTED constituent, quant à elles, une troisième catégorie qui regroupe des comités dont l'action de soutien est soit indispensable, soit complémentaire à l'existence même de l'Association. On y retrouve, entre autres, le comité des règlements, le comité d'organisation du congrès, le comité de rédaction de Documentation et bibliothèques et le comité des publications. Ce sont des comités permanents répondant aux impératifs de survie de I'Association.

Il est temps de cesser de fonctionner à la pièce. Jusqu'ici, tous les secteurs d'intervention de I'ASTED ont malheureusement été marqués de cette constante. Il est également temps de transcender les mandats des élus et d'inscrire toute action dans une perspective d'avenir. Cet objectif ne sera atteint que si suffisamment de membres se sentent concernés par cet avenir à réaliser. Car il est bon de se rappeler qu'une association est à l'image de ses membres et qu'une société a les gouvernants qu'elle veut bien se donner.

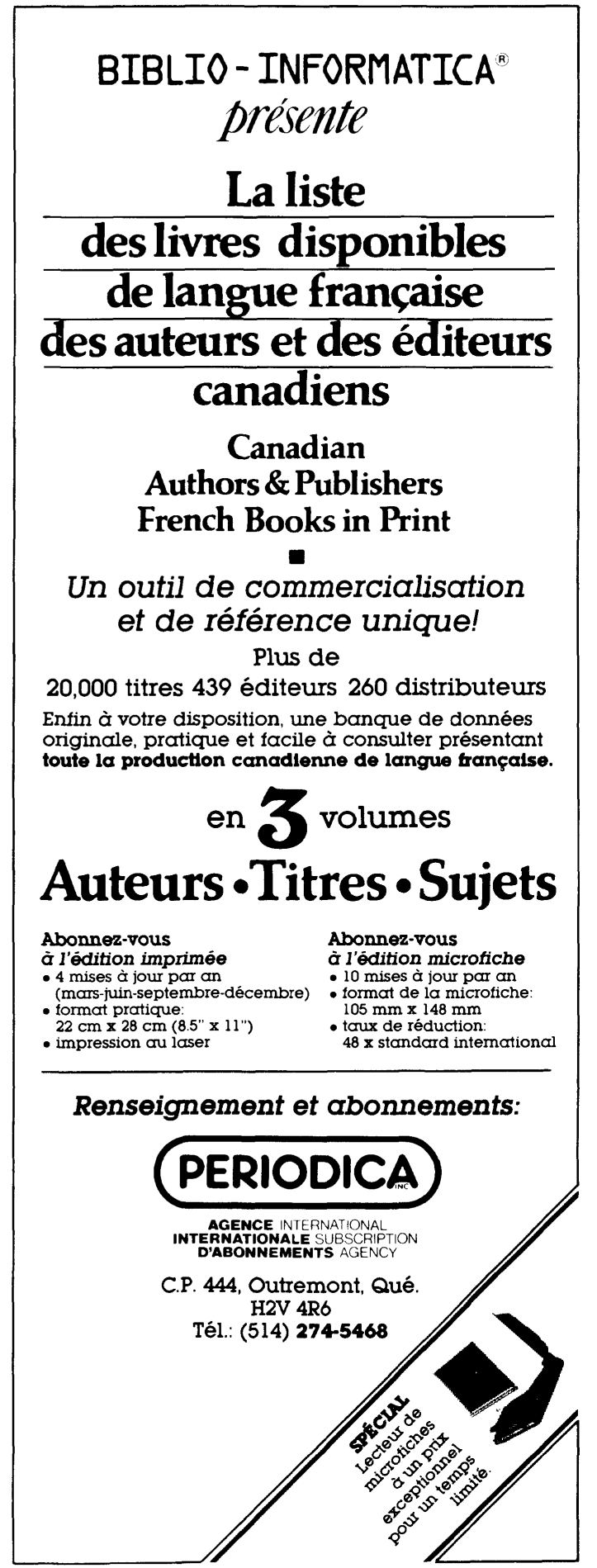

